\title{
The influence of lower limb plyometric and resistance training on the stiffness of Achilles and patellar tendons in recreational athletes
}

\author{
Khushpal Kaur Brar, Pavak Bhardwaj, Prabu Raja G \\ Department of Exercise and Sports Sciences, Manipal College of Health Professions, Manipal Academy of Higher \\ Education, Manipal, India
}

\begin{abstract}
Study aim: This study aimed to investigate the influence of combined plyometric and resistance training of lower limbs when administered for a shorter duration of six weeks on the stiffness of Achilles and patellar tendons as well as the jump height. Materials and methods: Twenty recreational athletes were administered six weeks of a single session of lower limb resistance training and one session of plyometric training every week for a total duration of six weeks. Tendon stiffness was measured using MyotonPro, and vertical jump height was derived from the force plate at baseline and six weeks after the intervention. Results: There was a statistically significant difference $(\mathrm{p}<0.01)$ between the baseline and post-training measures of patellar and Achilles tendons stiffness as well as the squat jump (SJ) and countermovement jump (CMJ) height.

Conclusion: Both resistance and plyometric training may be incorporated into the training session as combined training showed significant improvements in jump height and tendon stiffness after six weeks of combined RT and PT.
\end{abstract}

\section{Keywords: Weight training - Jump height - Jump training - Stretch-shortening cycle - Vertical jump performance}

\section{Introduction}

The rising demand for sports performance has instigated intense and long duration training resulting in an increased risk of overuse injuries in athletes. The peak force imparted to the athletes is about 12.5 times their body weight in activities like running or jumping [22]. Among numerous athletic injuries, tendinopathies of the patellar and Achilles tendons are common [19].

Tendons act as "mechanical buffers" and play a vital role in the absorption and transmission of force from muscle to bone [17]. Optimal tendon stiffness is essential for enhancing sports performance and preventing sports injuries [21]. Tendinopathies may alter the mechanical properties of tendons leading to altered force transmission, which may affect the sports performance $[1,9]$. The decreased stiffness of the tendons may result in excessive strain and are associated with a higher risk of sports injuries.

A systematic review has reported that the collagen of the tendons adapts to the mechanical loading during exercises. An increase in the tendon stiffness can be attributed to the alterations in the material properties or an increase in the cross-sectional area (CSA) $[2,20,28]$. A study that investigated the effects of plyometric training (PT) on Achilles tendon showed that there is a reduction in the dissipative properties and an increase in the stiffness without any changes in the CSA. However, Kjaer et al. reported that resistance training (RT) for 12 weeks resulted in a region-specific increase in the CSA, especially in the proximal and the distal part of the patellar tendon, suggesting tendon hypertrophy [12].

Another study that investigated the unilateral training program for plantar flexors (PT on one side of the lower limb and RT on the other side) showed the following changes. The RT increased the Achilles tendon (AT) stiffness but not joint stiffness. Conversely, PT increased the joint stiffness, but not the AT stiffness. Also, the PT increased squat jump (SJ) and countermovement jump (CMJ) heights effectively, when compared with RT [15]. Thus, combining RT and PT during the training session may improve the tendon and joint stiffness, thereby augmenting the sports performance.

A study showed that a minimum of three months of RT is required to observe an improvement in the tendon stiffness, whereas the stiffness decreases to the pre-training 
level within two months of detraining [13]. Although PT is shown to be effective in improving the vertical jump performances of countermovement and squat jumps, better improvements will be seen when done for a longer duration ( $\geq 10$ weeks) [27].

Nevertheless, Donald et al. demonstrated a significant improvement in the lower limb strength following either PT or RT when administered twice a week for just six weeks [18]. Thus, it is essential to investigate the effect of combined RT and PT on tendon stiffness and vertical jump performance when incorporated for a shorter duration. This study's objectives were to examine the influence of combined RT and PT on patellar and Achilles tendon stiffness as well as vertical jump performance when administered for six weeks.

\section{Material and methods}

\section{Study design}

A single group, pretest-posttest design was utilized.

\section{Study setting}

The study was conducted at the Centre for Sports Science, Medicine, and Research, Manipal College of Health Professions, Manipal Academy of Higher Education in India.

\section{Participants}

Twenty recreational athletes between the ages of 18 and 35 volunteered for the study. The inclusion criteria included the following. The participants were required to have a weight training history of at least three times per week for two months continuously in the past six months. In addition, the participants had to be able to perform a single-leg half-squat without pain and five repetitions of half-squat in 5 seconds with a load of $60 \%$ of body weight. Athletes with lower limb musculoskeletal pain or any medical conditions contraindicated for resistance training were excluded. Consequently 20 athletes were eligible (age: $22.1 \pm 3.81$ years; height: $171.3 \pm 8.53 \mathrm{~cm}$; weight $69.5 \pm 10.5 \mathrm{~kg}$; mean $\pm \mathrm{SD}$ ), including eighteen males and two females.

\section{Procedure}

After approval from the Institutional Research Committee, ethical clearance was obtained from the Institutional Ethics Committee (IEC 799/2019). The study was registered in the Clinical Trials Registry of India (CTRI/2019/12/022462). Each participant voluntarily provided written informed consent before participating. The procedure of the study is shown in Figure 1.

\section{Interventions}

All participants underwent 12 training sessions, including one session of RT and one session of PT in a week. There was an interval of a minimum of two days between the RT and PT sessions in a week. One familiarization session was given to all the participants before the training sessions.

Training protocol: A standardized warm-up, including jogging and dynamic stretching was provided for all participants before the beginning of each training session. The principal investigator supervised all sessions. The total duration of the session lasted for about 45 minutes to an hour.

The plyometric training program consisted of the following plyometric drills: two-foot ankle hop, squat jumps, box drills, and depth jumps, as shown in Table 1. The PT sessions' progression was made every two weeks during

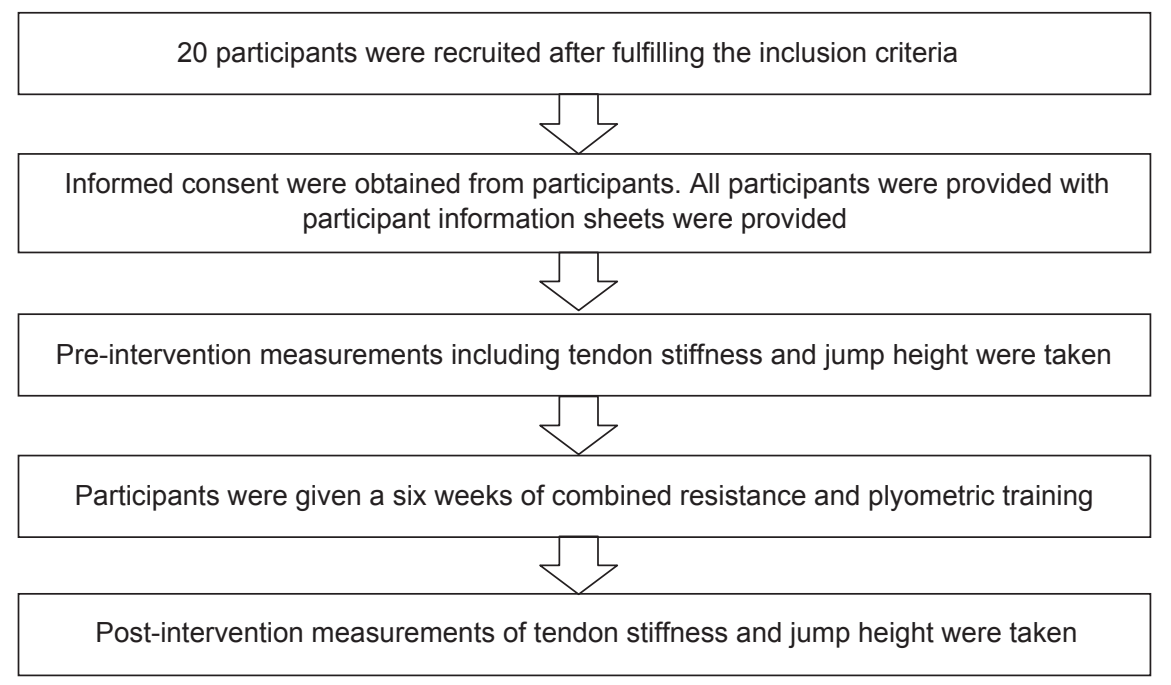

Figure 1. Study procedure 
Table 1. Interventions (Lower Limb Resistance training and Plyometric training)

\begin{tabular}{llll}
\hline Intervention & Exercises & Frequency (sets $\times$ reps $)$ & Rest period \\
\hline \multirow{2}{*}{$\begin{array}{l}\text { Lower limb } \\
\text { Plyometric training }\end{array}$} & Squat jump, & & \\
& Box drills & 2 sets $\times 10$ reps each & $2-3$ minutes \\
& Depth jump & & \\
\hline & Squat (high bar) & $1^{\text {st }}$ set $\times 12$ reps $\times 70 \%$ of $1 \mathrm{RM}$ & 3 minutes \\
Lower limb & Leg Press & $2^{\text {nd }}$ set $\times 10$ reps $\times 75 \%$ of $1 \mathrm{RM}$ & \\
Resistance training & Standing calf raise & $3^{\text {rd }}$ set $\times 8$ reps $\times 80 \%$ of $1 \mathrm{RM}$ & \\
& Seated calf raises & & \\
\hline
\end{tabular}

the six weeks of training sessions by increasing the height of the plyometric box. The first two weeks of PT sessions were done using a plyometric box of 22 inches, whereas, during the third and fourth weeks, 24 inches and the last two weeks, 26 inches were used. The rest period between sets was 2 to 3 minutes [9, 10, 23].

Testing: The resistance-training program is based on the one-repetition maximum (1RM), which was estimated using a 1RM estimation table [25]. Although 1RM testing is considered as the gold-standard procedure for the measurement of maximal muscular strength, considering the safety and effectiveness, ten-repetition maximum (10RM) testing was preferred in this study, from which $1 \mathrm{RM}$ was estimated. The procedure is similar to 1RM testing except for the inclusion of 10 repetitions. 10RM testing begins with a warm-up set consisting of 10 repetitions with $50 \%$ of the estimated 10RM load. The warm-up load was in the range of $30-40 \%$ of the participant's bodyweight. The 10RM was determined if the participant was able to complete the tenth repetition but unable to perform the eleventh repetition. If the volunteers were able to perform more than ten repetitions, the load was increased by $5-10 \%$. An interval of three minutes was given in-between the sets [24, 25].

Training: Exercises were performed according to the percentage of 1RM. The weight-training program included four exercises: high bar squat, leg press, seated, and standing calf raises. The frequency of training was three sets of each exercise per session. The intensity varied from $67 \%$ to $80 \%$ of $1 \mathrm{RM}$. The rest period between the sets was 3 minutes, as shown in Table 1.

\section{Outcome measures}

The outcome measures include measurement of the stiffness of Achilles and patellar tendons using Myoton Pro and the vertical jump performance by measuring jump height using a force plate. The outcome assessor was blinded.

The MyotonPro digital palpation device (Myoton AS, Estonia and Myoton Ltd, London) was pre-calibrated by the manufacturer and was done a month prior to the assessments. The MyotonPro digital palpation device was connected with Myoton AS software (Version 5.0.0) for attaining the required data $(\mathrm{ICC}=0.96)$. The MyotonPro device was used to measure the stiffness of Achilles and patellar tendons in newtons/meter $(\mathrm{N} / \mathrm{m})$. The measurement is based on the free oscillation technique, where the probe of the device was placed perpendicular to the skin. A brief mechanical impulse was applied, resulting in damped oscillation of the tissue, which was recorded. The obtained filtered acceleration curves were used to calculate the viscoelastic properties of the tissue. The stiffness can be inferred from the filtered curve as follows. Stiffness $=\mathrm{a} 0 \times \mathrm{m}_{\text {probe }} / \Delta \mathrm{l}$, where $\mathrm{a} 0$ is the first negative peak (maximal acceleration of the curve), $\mathrm{m}_{\text {Probe }}$ is the mass of the probe (probe), and $\Delta \mathrm{l}$ is the maximal displacement of the tissue [8]. The device was placed $4 \mathrm{~cm}$ above the calcaneal tuberosity in prone lying for the Achilles tendon and at the insertion of the patellar tendon in sitting, as shown in Figure 2 [26]. Three measurements were taken for both patellar and Achilles tendons and the average of these stiffness measures was considered and expressed in newtons/meter $(\mathrm{N} / \mathrm{m})$.

\section{AMTI's Biomechanics Force Platform (AMTI, Inc. Watertown, MA)}

The force platform was interfaced with AMTI's Net force software (Version 3.5.3), used for data acquisition, and AMTI's Bioanalysis software (Biosoft, Version 2.3.2) was used for data analysis $(\mathrm{ICC}=0.98)$. Jump height measurement was taken on a digital platform force plate as shown in Figure 3. The squat jump (SJ) and countermovement jumps (CMJ) were performed three times for both the jumps and the average of these values was considered. Data were transferred to software and jump height calculation was done using flight time $[5,6,16]$.

\section{Data analyses}

The recorded data were analyzed using SPSS version 16. Descriptive statistics were used to measure 


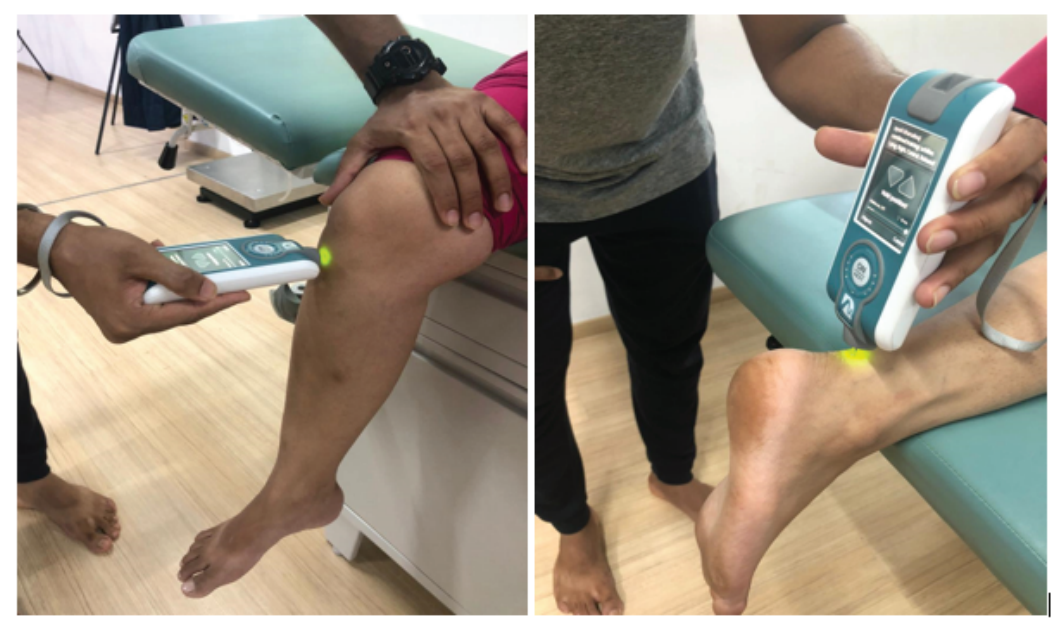

Figure 2. Measurement of Patellar and Achilles tendon stiffness using Myoton Pro
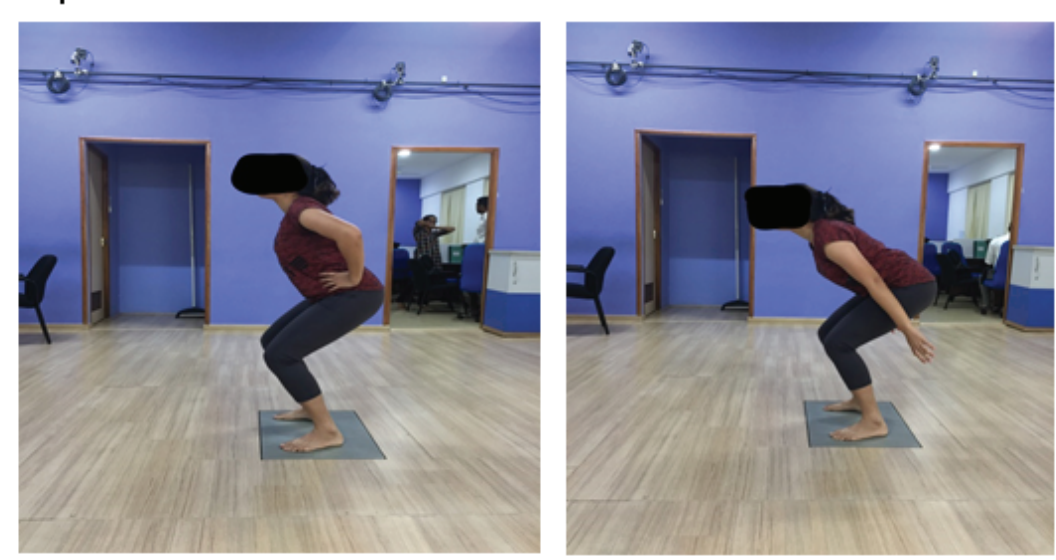

Figure 3. Measurement of Squat and Countermovement jump height using a force plate

baseline demographic data. The Kolmogorov-Smirnov test showed that data for patellar tendon stiffness and countermovement jump height followed the normal distribution; thus, the paired t-test was used to analyze the differences following interventions. The Wilcoxon signed rank test was used to identify the differences in the AT stiffness and SJ height with the level of significance $\mathrm{p} \leq 0.05$.

\section{Results}

The Wilcoxon-signed rank test showed a statistically significant difference $(\mathrm{p}<0.01)$ between the pre- and posttest measurements of AT stiffness and SJ height as shown in Table 2. The paired ' $t$ ' test that was used for the analysis for PT stiffness and CMJ height showed a statistically

Table 2. Comparison of Achilles tendon stiffness and SJ height following training

\begin{tabular}{lccccc}
\hline & $\begin{array}{c}\text { Baseline } \\
\text { Median (IQR) }\end{array}$ & $\begin{array}{c}\text { Post training } \\
\text { Median (IQR) }\end{array}$ & Difference [\%] & Significance & Effect size r \\
\hline AT stiffness left $[\mathrm{N} / \mathrm{m}]$ & $839(771,910)$ & $946(903,1011)$ & 12.8 & $\mathrm{p}<0.01$ & $>0.5$ \\
AT stiffness right $[\mathrm{N} / \mathrm{m}]$ & $850(792.5,930.5)$ & $946(896.5,1030)$ & 11.3 & $\mathrm{p}<0.01$ & $>0.5$ \\
SJ Height $[\mathrm{cm}]$ & $28.5(25.25,32.76)$ & $33.5(31,37.5)$ & 17.5 & $\mathrm{p}<0.01$ & $>0.5$ \\
\hline
\end{tabular}


Table 3. Comparison of patellar tendon stiffness and CMJ height following training

\begin{tabular}{lccccc}
\hline & $\begin{array}{c}\text { Baseline } \\
\text { Mean } \pm \mathrm{SD}\end{array}$ & $\begin{array}{c}\text { Post training } \\
\text { Mean } \pm \mathrm{SD}\end{array}$ & Difference [\%] & Significance & $\begin{array}{c}\text { Effect size } \\
\text { Hedge's g }\end{array}$ \\
\hline PT stiffness left $[\mathrm{N} / \mathrm{m}]$ & $809.5 \pm 118$ & $935 \pm 71.7$ & 15.5 & $\mathrm{p}<0.01$ & $>0.8$ \\
PT stiffness right $[\mathrm{N} / \mathrm{m}]$ & $782.35 \pm 125.5$ & $927 \pm 74.2$ & 18.5 & $\mathrm{p}<0.01$ & $>0.8$ \\
CMJ Height $[\mathrm{cm}]$ & $32.95 \pm 4.8$ & $36.95 \pm 4.8$ & 12.1 & $\mathrm{p}<0.01$ & 0.8 \\
\hline
\end{tabular}

PT - Patellar tendon, CMJ - Countermovement jump,

significant difference in baseline and post-training measures as shown in Table 3.

\section{Discussion}

Although remodeling of the tendon is considered to occur in response to strength or endurance training, there is inconsistent information regarding the influence of RT on the mechanical properties of the tendon [4]. The increase in the stiffness of tendons is considered necessary in preventing injuries such as tendinopathies in athletes. Stiffness of Achilles and patellar tendons may play an essential role in vertical jump performance besides joint stiffness. An increase in the stiffness of tendons can influence the muscle's ability to rapidly generate force. Long-term resistance training results in an increase in the total number as well as the diameter of collagen fibrils [3]. Plyometric training when administered for 12 weeks showed improvement in the stretch-shortening cycle exercise performance by optimizing the muscle-tendon behavior and increasing the tendon stiffness [11]. Although RT and PT have been shown to improve the tendon stiffness and jump height, it usually takes more than ten weeks of training sessions when administered individually. This study intended to investigate the influence of combined RT and PT of lower limbs for just six weeks on the stiffness of Achilles and patellar tendons as well as countermovement and squat jump height. There was a significant improvement in the stiffness of Achilles and patellar tendons as well as jump height following six weeks of resistance and plyometric training.

A study reported that isometric training for 12 weeks not only increased muscle strength and size but also increased the stiffness and Young's modulus of the human tendon of the vastus lateralis [14].

Kubo et al. investigated the effects of unilateral plyometric and resistance training on the muscle-tendon complex of plantar flexors and jump performance. The RT increased the Achilles tendon (AT) stiffness but not joint stiffness. On the other hand, PT increased joint stiffness, but not the AT stiffness. Also, the PT increased SJ and CMJ heights effectively, when compared with RT [15]. Thus, including both RT and PT sessions may improve the tendon and joint stiffness, thereby aiding in augmenting the sports performance.

Incorporating RT and PT during the athletic training sessions may be useful in improving the tendon stiffness and vertical jump performance even when performed for a shorter duration of 6 weeks, which signifies the role of combined PT and RT in performance enhancement. Thus, incorporating both RT and PT during the athletic training sessions may be useful in improving the tendon stiffness and vertical jump performance even when performed for a shorter duration of six weeks. The results of the study have key implications for coaches in planning the training protocols for athletes involved in explosive sports focusing on enhancing sports performance with less likelihood of injuries.

Conflict of interest: Authors state no conflict of interest.

\section{References}

1. Aicale R., Tarantino D., Maffulli, N. (2018) Overuse injuries in sport: A comprehensive overview. J. Orthop. Surg. Res., 13(1): 309. DOI: 10.1186/s13018-018-1017-5.

2. Bohm S., Mersmann F., Arampatzis A. (2015) Human tendon adaptation in response to mechanical loading: a systematic review and meta-analysis of exercise intervention studies on healthy adults. Sports Medicine Open, 1(1): 7. DOI: 10.1186/s40798-015-0009-9.

3. Brumitt J., Cuddeford T. (2015) Current concepts of muscle and tendon adaptation to strength and conditioning. Int. J. Sports Phys. Ther, 10(6): 748-759. PMID: 26618057; PMCID: PMC4637912.

4. Buchanan C.I., Marsh R.L. (2002) Effects of exercise on the biomechanical, biochemical, and structural properties of tendons. Comparative biochemistry and physiology. Part A, Mol. Integr. Physiol., 133(4): 1101-1107. DOI: 10.1016/s1095-6433(02)00139-33. 
5. Buckthorpe M., Morris J., Folland J.P. (2012) Validity of vertical jump measurement devices. J. Sports Sci., 30(1): 63-69. DOI: 10.1080/02640414.2011.624539.

6. Cheah P.Y., Cheong J.P.G., Razman R., Zainal Abidin N.E. (2017) Comparison of Vertical Jump Height Using the Force Platform and the Vertec. $3^{\text {rd }}$ International Conference on Movement, Health and Exercise. MoHE. IFMBE proceedings, Vol. 58. Springer, Singapore. DOI: 10.1007/978-981-10-3737-5_33.

7. Couppé C., Kongsgaard M., Aagaard P., Hansen P., Bojsen-Moller J., Kjaer M., Magnusson SP. (2008) Habitual loading results in tendon hypertrophy and increased stiffness of the human patellar tendon. J. Appl. Physiol., (Bethesda, Md.: 1985), 105(3): 805-810. DOI: 10.1152/ japplphysiol.90361.2008.

8. Cristi-Sánchez I., Danes-Daetz C., Neira, A., Ferrada W., Yáñez Díaz R., Silvestre Aguirre R. (2019) Patellar and Achilles Tendon Stiffness in Elite Soccer Players Assessed using Myotonometric Measurements. Sports Health, 11(2): 157-162. DOI: 10.1177/1941738118820517.

9. Davies G., Riemann B.L., Manske R. (2015) Current Concepts of Plyometric Exercise. Int. J. Sports Phys. Ther, 10(6): 760-786. PMID: 26618058; PMCID: PMC4637913.

10. Fouré A., Nordez A., Cornu C. (2012) Effects of plyometric training on passive stiffness of gastrocnemii muscles and Achilles tendon. Eur. J. Appl. Physiol., 112(8): 2849-2857. DOI: 10.1007/s00421-011-2256-X.

11. Hirayama K., Iwanuma S., Ikeda N., Yoshikawa A., Ema R., Kawakami Y. (2017) Plyometric Training Favors Optimizing Muscle-Tendon Behavior during Depth Jumping. Front. Physiol., 8: 16. DOI: 10.3389/ fphys.2017.00016.

12. Kjaer M., Langberg H., Heinemeier K., Bayer M.L., Hansen M., Holm L., Magnusson S.P. (2009) From mechanical loading to collagen synthesis, structural changes, and function in human tendon. Scand. J. Med. Sci. Sports, 19(4): 500-510. DOI: 10.1111/j.1600-0838.2009.00986.x.

13. Kubo K., Ikebukuro T., Yata H., Tsunoda N., Kanehisa H. (2010) Time course of changes in muscle and tendon properties during strength training and detraining. J. Strength Cond. Res., 24(2): 322-331. DOI: 10.1519/ JSC.0b013e3181c865e2.

14. Kubo K., Kanehisa H., Ito M., Fukunaga T. (2001) Effects of isometric training on the elasticity of human tendon structures in vivo. J. Appl. Physiol., (Bethesda:1985), 91(1): 26-32. DOI: 10.1152/jappl.2001.91.1.26.

15. Kubo K., Morimoto M., Komuro T., Yata H., Tsunoda N., Kanehisa H., Fukunaga T. (2007) Effects of plyometric and weight training on muscle-tendon complex and jump performance. Med. Sci. Sports Exerc., 39(10): 1801-1810. DOI: 10.1249/mss.0b013e31813e630a.
16. Linthorne N.P. (2001) Analysis of standing vertical jumps using a force platform. Am. J. Phys., 69: 1198. DOI: 10.1119/1.1397460.

17. Liu C.L., Li Y.P., Wang X.Q., Zhang ZJ. (2018) Quantifying the Stiffness of Achilles Tendon: Intra- and Inter-Operator Reliability and the Effect of Ankle Joint Motion. Medical Science Monitor: International Medical Journal of Experimental and Clinical Research, 24: 4876-4881. DOI: 10.12659/MSM.909531.

18. MacDonald C.J., Lamont H.S., Garner J.C. (2012) A comparison of the effects of 6 weeks of traditional resistance training, plyometric training, and complex training on measures of strength and anthropometrics. J. Strength Cond. Res., 26(2): 422-431. DOI: 10.1519/ JSC.0b013e318220df79.

19. Mascaró A., Cos M.À., Morral A., Roig A., Purdam C., Cook J. (2018) Load management in tendinopathy: Clinical progression for Achilles and patellar tendinopathy. Apunts. Medicina de l'Esport, 53(197): 19-27. DOI: 10.1016/j.apunts.2017.11.005.

20. Miller B.F., Olesen J.L., Hansen M., Dossing S., Crameri R.M., Welling R.J., Rennie M.J. (2005) Coordinated collagen and muscle protein synthesis in human patella tendon and quadriceps muscle after exercise. J. Physiol., 567(Pt 3): 1021-1033. DOI: 10.1113/ jphysiol.2005.093690.

21. Morgan G.E., Martin R., Williams L., Pearce O., Morris K. (2018). Objective assessment of stiffness in Achilles tendinopathy: a novel approach using the MyotonPRO. BMJ Open Sport Exerc. Med., 4(1): e000446. DOI: 10.1136/bmjsem-2018-000446.

22. Paavola M., Kannus P., Järvinen M. (2005) Epidemiology of Tendon Problems in Sport. In: Maffulli,N., Renström P, Leadbetter W.B (eds.), Tendon Injuries. Springer, London. DOI: 10.1007/1-84628-050-8_5.

23. Potach D.H., Chu D.A. (2016) Chapter 18 - Program design and technique for plyometric training taken from Essentials of strength training and conditioning. ISBN: 978-0-203-85104-3, Vol. 4: 471-513.

24. Santos W., Siqueira G., Martins W.R., Vieira A., Schincaglia R.M., Gentil P., Vieira C.A. (2019) Reliability and Agreement of the 10-Repetition Maximum Test in Breast Cancer Survivors. Front. Oncol., 9: 918. DOI: 10.3389/ fonc.2019.00918.

25. Sheppard J.M., Triplett N.T. (2016) Chapter 17 - Program design for resistance training taken from Essentials of strength training and conditioning. ISBN: 978-0-20385104-3, Vol. 4, pp. 451-459.

26. Sohirad S., Wilson D., Waugh C., Finnamore E., Scott A. (2017) Feasibility of using a hand-held device to characterize tendon tissue biomechanics. PloS one, 12(9): e0184463. DOI: 10.1371/journal.pone.0184463. 
27. Stojanović E., Ristić V., McMaster D.T., Milanović Z. (2017) Effect of Plyometric Training on Vertical Jump Performance in Female Athletes: A Systematic Review and Meta-Analysis. Sports Med., (Auckland, N.Z.), 47(5): 975-986. DOI: 10.1007/s40279-016-0634-6.

28. Wiesinger H.P., Kosters A., Muller E., Seynnes O.R. (2015) Effects of increased Loading on in Vivo Tendon Properties: A Systematic Review. Med. Sci. Sports Exerc., 47(9): 1885-1895. DOI: 10.1249/MSS.0000000000000603.

\section{Received 05.06.2020}

Accepted 10.12.2020

(C) University of Physical Education, Warsaw, Poland

Acknowledgments

The authors would like to thank all the study participants and Ms. Shruti Shenoy for editing the manuscript. 